\title{
Research on Atmospheric Density Online Measurement Technology Based on Spacecraft Platform
}

\author{
Chaojie Wang and Xiao Li \\ National university of defense technology \\ China \\ National Key Laboratory of Science and Technology on \\ Nearspace Vehicle Technology \\ China \\ Chunlei Li \\ The Party School of Jilin Province \\ China
}

\author{
Lai'an Qin* \\ Anhui Institute of Optics and Fine Mechanics \\ China \\ ${ }^{*}$ Corresponding author
}

\begin{abstract}
It is necessary to implement online measurement of atmospheric density around spacecraft in order to better design aerodynamic appearance of hypersonic spacecraft and meet the demand of pneumatic identification. This is one of important problems that should be solved in domestic spacecraft design field but cannot be solved. In the paper, a technical solution of measuring atmospheric density by imaging lidar based on Rayleigh scattering principle is proposed aiming at flight characteristics of spacecraft and surrounding environment features during flight, atmospheric density online measuring instrument is developed, and difficulties and innovations in the development process are introduced. Calibration results indicate that precision of atmospheric density online measurement with the instrument can be controlled within 5\%. Successful development of the instrument provides a new method for designing spacecraft appearance and improving aerodynamic identification.
\end{abstract}

Keywords-atmospheric density; online measurement; lidar; spacecraft; Rayleigh scattering

\section{INTRODUCTION}

Aerodynamic characteristics of spacecraft should be studied in order to ensure normal flight of hypersonic spacecraft. The aerodynamic characteristics of spacecraft should be represented by dimensionless aerodynamic characteristic parameters (lift coefficient, drag coefficient, pitching moment coefficient, etc.) according to the similarity law theory. They are defined as follows: Aerodynamic force/ (flying action pressure*reference area) (or aerodynamic torque/ (flying action pressure*reference area* reference length). Therefore, both aerodynamic forces or aerodynamic moment and accurate value of flying action pressure are required for identifying aerodynamic characteristic parameters according to flying test data. Flying action pressure is defined as multiplying atmospheric density and flying speed square, and the result is divided by 2 . Therefore, accurate access to local atmospheric density has important significance for pneumatic identification. It is greatly difficult to implement accurate aerodynamic identification at high altitude since atmosphere density is fluctuated greatly and it is not easy to measure at high altitude.

Currently, measurement of static atmospheric density has been massively studied at home and abroad. Main developed measuring instruments include atmospheric density detector, incoherent scattering lidar, mass spectrometer, etc. [1-3]. Atmospheric density detector is used for obtaining free atmospheric density through directly detecting gas pressure and temperature in sensor, and combining with basic relation formula established by theory of gas molecular dynamics. Active gas calibration system is respectively used for calibrating and rechecking the detector respectively with standard gas sample O2, He, etc. on the ground. The method cannot be applied to online measurement of high altitude and high speed environment. Incoherent scattering lidar, mass spectrometer and other remote sensing measurement methods are mostly used for measurement under foundation measurement static environment and measurement under low speed dynamic environment due to limitation of own measurement system. It is difficult to obtain high temporal and spatial resolution and high precision atmospheric density data under hypersonic flight environment.

In the paper, active Rayleigh scattering imaging measurement method based on EMCCD is proposed aiming at demand of online measurement of atmospheric density on hypersonic spacecraft. Rayleigh scattering light intensity of gas molecules is proportional to the molecular density [4-6]. The feature is utilized for obtaining high precision online atmospheric density data at different distances through analyzing laser backward Rayleigh scattering light cross in the flow field area photographed by EMCCD. 


\section{BASIC PRINCIPLE OF ATMOSPHERIC DENSITY MEASUREMENT}

Rayleigh scattering imaging is based on measurement method of molecular optics in flow field. The method not only is suitable for the flow field measurements of gas molecules, but also has unique advantages in the aspect of measuring plasma flow field. Therefore, it is especially suitable for measurement under high speed condition, such as measurement of hypersonic, high temperature and high enthalpy plasma flow fields. It is free from the problem of dependence on measuring wavelength and substance.

Aerosol content is minimal in flight area of hypersonic spacecraft, especially during flight in near space. Atmosphere in the measurement area can be regarded as clean atmosphere without aerosols. Atmospheric echo received by imaging lidar mainly includes scattering signals generated by atmospheric molecules. Molecule scattering in gas flow field can be handled by Rayleigh scattering theory. If the effective light field of light wave acted on the molecule is $E$, radiation intensity $I$ on direction $\mathrm{H}$ can be expressed as follows[6] under the condition that $\mathrm{N}$ gas modules are randomly distributed and isotropic with diameter much shorter than working wavelength.

$$
I=\frac{8 \pi N e^{4} E^{2}}{M^{2} k_{0}^{4} \lambda^{4}}\left(1+\cos ^{2} H\right)
$$

Wherein, e refers to quantity of electricity; $M$ refers to particle mass; $\mathrm{k}_{0}$ refers to dipole inherent frequency.

Formula (1) shows that Rayleigh scattering light intensity is proportional to gas molecule number density of gas. Therefore, spatial distribution of atmospheric density can be retrieved through analyzing back-scattering signal intensity.

Principle of Rayleigh scattering imaging lidar designed in the paper is shown in Figure I. The lidar equation of the laser can be expressed as follows:

$$
E_{r}=\frac{K E_{l} A T_{a t m z} T_{a t m R} \beta(\theta, \Phi, z) d z}{R^{2}}
$$

Wherein, Er refers to energy received by unit pixel at height $\mathrm{z}$ and viewing angle $\mathrm{d} \theta$. R refers to the distance from CCD to $\mathrm{dz} ; \mathrm{E}_{1}$ refers to total energy of lasing within exposure time; A refers to effective receiving aperture. $\mathrm{T}_{\text {atmz }}$ refers to atmospheric transmittance from laser to height $\mathrm{z} ; \mathrm{T}_{\mathrm{atmR}}$ refers to atmospheric transmittance from height $\mathrm{z}$ to EMCCD camera; $\beta(\theta, \Phi, z)$ is scattering coefficient, which is associated with scattering angle $\theta$, polarization angle $\Phi$ and height $\mathrm{z}$; dz refers to distance resolution corresponding to light spot image unit length; $\mathrm{K}$ refers to calibration constant of the lidar. Atmospheric transmittance change of $532 \mathrm{~nm}$ wavelength laser from $30 \mathrm{~km}$ to $80 \mathrm{~km}$ is less than $0.5 \%$ according to simulation calculation results with moderate resolution atmospheric radiation transmission software Modtran. Therefore, atmospheric transmittance can be used as a constant in the lidar system due to shorter measurement distance.

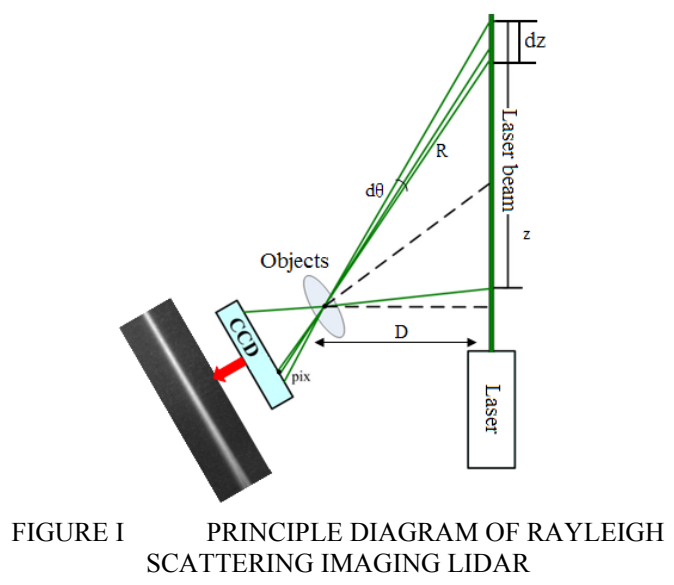

The follows can be obtained according to geometrical relationship:

$$
d z=\frac{R^{2} d \theta}{D}
$$

The follows can be obtained by bringing formula (3) to formula (2):

$$
E_{r}=\frac{K E_{l} A T_{a t m z} T_{a t m R} \beta(\theta, \Phi, z) d \theta}{D}
$$

Influence of high altitude aerosol is ignored, photon echo received by imaging lidar basically belongs to molecular Rayleigh scattering signal, and the atmospheric attenuation in the measurement area is tiny. Since atmospheric molecule scattering can be regarded as independent scattering, light scattering coefficient of unit volume atmospheric molecules is the product of single atmospheric molecule light scattering cross section and atmospheric molecular number density $n$. Therefore, the equation (4) can be expressed as follows:

$$
E_{r}=\frac{K E_{l} A T_{a t m z} T_{a t m R} n(z) \sigma_{\text {Ray }} d \theta}{D}
$$

Wherein, $\sigma_{\text {Ray }}$ is Rayleigh back-scattering cross section of $532 \mathrm{~nm}$ wavelength.

The calculation formula of atmospheric density at different heights can be expressed with the following formula according to the relationship between atmospheric density and atmospheric molecular number density $\mathrm{n}$ :

$$
\rho(z)=\frac{n(z) k}{R}=\frac{E_{r} D k}{K E_{l} A T_{a t m z} T_{a t m R} \sigma_{\text {Ray }} d \theta R}
$$

In the formula, $\mathrm{k}$ is the boltzmann constant, and $\mathrm{R}$ is the molar gas constant. 
Laser echo image is composed of signal light column and background. Points of light column at different positions correspond to atmospheric density distribution at different heights. Image processing, averaging and denoising are implemented on collected laser echo images. Atmospheric density at different heights can be obtained finally according to calculation with formula (6).

\section{AtMospheric Density MEASUREMENT SCHEME}

Since Rayleigh scattering does not have selectivity on laser wavelength, Rayleigh scattering light intensity of gas molecules is proportional to the molecular density. The strength of Rayleigh scattering image reflects the atmospheric density distribution. Therefore, principle of Rayleigh scattering is adopted in the Scheme and it is realized by lidar system.

\section{A. Composition of Atmospheric Density Online Measuring Instrument}

An active lidar remote sensing technology is adopted in atmospheric density online measurement. It is a product by combining laser technology and lidar technology. The technology has advantages of wide measuring scope, high measurement precision, high spatial and temporal resolution, continuous measurement, etc. System structure diagram is shown in Figure II. The laser outputs laser beam with $532 \mathrm{~nm}$ wavelength, which is vertically projected in atmosphere. The laser beam produces Rayleigh scattering due to effect of air molecules in the atmosphere. A part of scattering light produced by Rayleigh scattering effect is collected by detector, and the collected echo signal reflects atmospheric molecule density. The density is higher, echo signal is stronger.

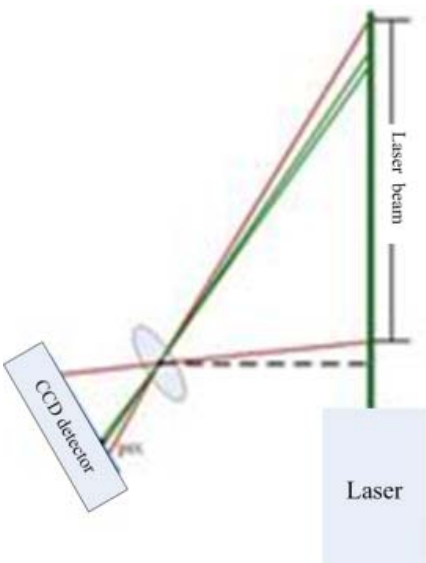

FIGURE II SCHEMATIC DIAGRAM OF ATMOSPHERIC DENSITY ONLINE INSTRUMENT SYSTEM STRUCTURE

Atmospheric density online measuring instrument structure can be divided into three parts of laser emission, optical receiving as well as data acquisition and control (electronics). Laser transmitting part and receiving part are separated. Laser emission part consists of solid-state laser, light beam collimation parts and light guiding mirrors. The energy meter should be used to monitor the energy of emitting laser. Laser beam splitter is installed in front of the laser, and a small part of light is separated for energy meter. Optical receiving part is mainly composed of EMCCD camera and receiving lens. Appropriate objective is used for including the measuring range into EMCCD camera view field through geometric structure design. Narrow bandpass filter is installed between the lens and EMCCD in order to eliminate stray light disturbance such as plasma, etc. Embedded system is adopted in data collection and control part. Control, data acquisition, processing, storage and transmission can be realized through software.

Technical indicators of atmospheric density online measuring instrument are finally designed as shown in table 1 according to actual demand of hypersonic spacecraft on atmospheric density online measurement.

TABLE I MAIN TECHNICAL INDICATORS OF RAYLEIGH SCATTERING IMAGING LIDAR

\begin{tabular}{|l|l|}
\hline Main Parameters & Technical Indicators \\
\hline Working laser wavelength & $532 \mathrm{~nm}$ \\
\hline Laser power & $2 \mathrm{~W}$ \\
\hline Receiving Objective aperture & $30 \mathrm{~mm}$ \\
\hline System focal length & $50 \mathrm{~mm}$ \\
\hline Sending and receiving angle & $9^{\circ}$ \\
\hline Narrow band filter bandwidth & $0.1 \mathrm{~nm}$ \\
\hline Measurement area & $20 \mathrm{~mm}-3000 \mathrm{~mm}$ \\
\hline
\end{tabular}

\section{B. Software Design}

The system software is divided into three parts of EMCCD control, laser control and power control, wherein power control program can set start time or start to receive external control for certain time delay. Power make-and-break of all instruments can be controlled. EMCCD control and laser control mainly include parameter setting, data acquisition, data processing, etc. Software flow chart is shown in Figure III.

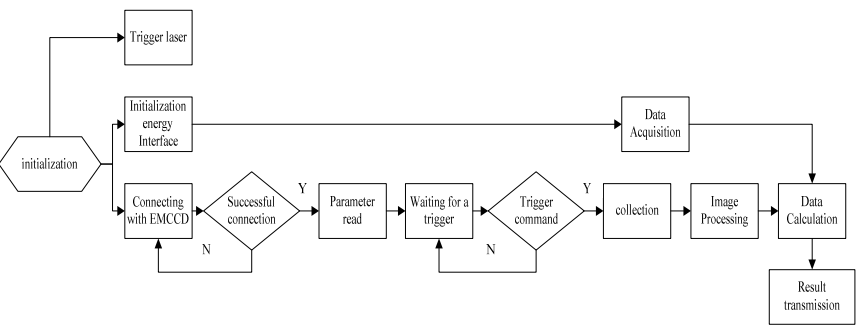

FIGURE III SOFTWARE FLOW CHART

Data and external communication are divided into two modes. One is transferring original image with certain time interval after being compressed.The other mode is transferring final density data after image processing.

\section{Calibration Conditions of Atmospheric Density Online Measuring Instrument}

Atmospheric density online measuring instrument must be calibrated before use in order to meet the demand of aerospace engineering. Currently, dynamic calibration during flight of spacecraft cannot be achieved. Flight state can be simulated on the ground for static calibration. Calibration should be implemented in pure air in order to avoid influence of aerosols in the atmosphere on calibration precision. The calibration process can be completed with ground vacuum pipeline [9]. The vacuum pipeline used for calibration is shown in Figure $\mathrm{IV}(\mathrm{a})$. Vacuum pipeline is 9 meters long with diameter of 0.5 
meters. The inside space of the pipeline is vacuumed and can be filled with nitrogen. Nitrogen pressure in the pipeline should be controlled through charging valve and exhaust valve, thereby realizing continuous adjustment of nitrogen density in the pipeline. Pressure value in the pipeline and temperature can be measured online. Measurement instrument is shown in Figure IV(b). Pressure measurement error is $\pm 0.5 \%$. Temperature measurement error is $\pm 1 \%$. Laser incident window has diameter of $100 \mathrm{~mm} .532 \mathrm{~nm}$ antireflection coating is coated on both sides, and the laser transmittance is higher than $99.9 \%$.

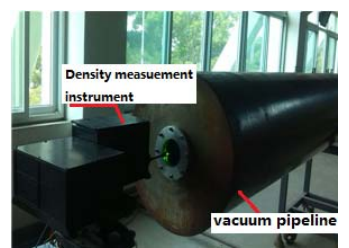

(A)

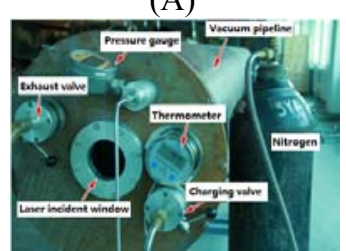

(B)

FIGURE IV CALIBRATION OF VACUUM PIPELINE:(A) VACUUM PIPELINE FRONT END SURFACE VIEW;(B) VACUUM PIPELINE REAR END SURFACE SENSOR DISTRIBUTION DIAGRAM

Nitrogen density in the pipeline is changed through charging valve during calibration. Atmospheric density online measuring instrument can be used for measuring a group of calibration data to analyze nitrogen densities in the pipeline under the condition. Meanwhile, reading of the thermometer and pressure gauge should be utilized for calculating theory value of gas density in the pipeline under the condition according to atmospheric state equation, and the results can be compared with measurement value. Pressure in the pipeline can be constantly changed for calibration. A series of measurement values can be obtained and compared with theoretical values.

\section{Analysis on Calibration Result of Atmospheric Density Online Measuring Instrument}

Laser emitted by atmospheric density online measuring instrument is input from the front incidence window of the vacuum pipeline. Back-scattering light can be received and imaged by optical receiving system through front end window. The collected images are shown in Figure V.
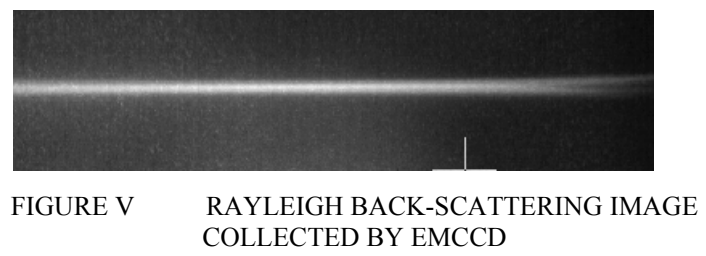

A series of density increasing and decreasing calibrations can be implemented under cooperation of vacuum pump in order to calibrate measurement precision of the instrument under different gas density environments. Gas density calibration value $\left(\mathrm{g} / \mathrm{m}^{3}\right)$ can be obtained by analyzing and calculating the collected image data. The values can be compared with gas density theoretical calculation value $\left(\mathrm{g} / \mathrm{m}^{3}\right)$ calculated from temperature and gas pressure. Temperature and gas pressure are measured by pipeline monitoring instrument. The results are shown in Figure VI.
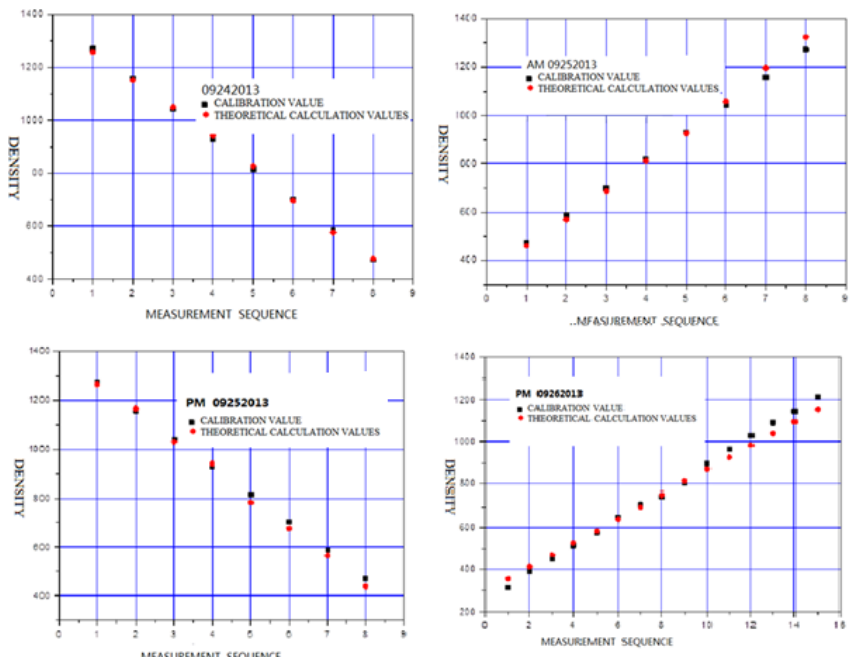

FIGURE VI COMPARISON BETWEEN GAS DENSITY CALIBRATION VALUE AND THEORETICAL CALCULATION VALUES

Each measurement sequence value in Figure VI represents one density sampling point. The figure shows that data obtained from measurement on April 24, 25 and 26, 2013 for many times is more stable. In addition, the values are consistent with theoretical values obtained according to atmospheric pressure and temperature. Wherein, errors between the instrument calibration values (in the morning of April 24 and 25 ) and theoretical calculation value are respectively $1.1 \%$ and $2.4 \%$ only. Error between instrument calibration value (in the afternoon of April 25 and at the evening of April 26) and the theoretical calculation value is larger, respectively $3.5 \%$ and $3.77 \%$. Though calculation error of data measured at different time sections is fluctuated to certain extent, the general measurement error of designed atmospheric density online measuring instrument can be controlled within 5\% through massive repeated calibrations and verifications.

\section{CONCLUSION}

In summary, atmospheric density online measuring instrument developed in the paper is used for solving spatial resolution of detected target through pixel resolution of image sensor. Measurement without blind area can be basically realized. Measurement of non-contact, high temporal resolution, high spatial resolution and high precision can be realized. Measurement precision can be better than 5\%. It overcomes defects of lidars with other detection structures, which cannot be applicable for atmospheric density online measurement with short distance and high spatial resolution. A new measurement method is proposed for online measurement of atmospheric density parameters around atmospheric spacecraft. It has been 
determined that the instrument will be used for some important model in domestic aerospace.

\section{REFERENCES}

[1] Luo Zhiyong, Liu Ziyong, Liu Jiping etc. "Development of portable air density precision measuring instrument," Journal of Measurement, vol.22, pp.52-56,2001.

[2] Mei Bing. "Analysis on empirical orthogonal function of incoherent scattering radar ionospheric electron density profile observation data", Wuhan, Science Master Theses of Institute of Geology and Geophysics, Chinese Academy of Sciences, 2007.

[3] Yang Ziqin. "Research on Aerosol particle density measurement method," Qufu, Master Thesis of Qufu Normal University, 2014.

[4] Wang Jie, Yao Jianquan, Yu Yizhong, etc. "Application of Rayleigh scattering for gas flow field two-dimensional transient density measurement," Journal of Electronics, Laser, vol.12, pp.62-64,2001.

[5] Wang Jie, Shi Xiangchun, Xiao Xuhui etc. "Application of Rayleigh scattering in molecular flow field multi-parameter measurement," Journal of Tianjin University, vol.1, pp.21-24,2000.

[6] Wang Jie, Shi Xiangchun, Li Xifu. "Application of Rayleigh scattering/laser induced fluorescence technique in air and $\mathrm{O} 2$ flow field two-dimensional transient measurement," Journal of Optics, vol.12, pp.1375-1380,1999. 